\title{
EXISTENCE OF CLASSICAL SOLUTIONS OF THE EQUATIONS OF MOTION FOR COMPRESSIBLE FLUIDS OF SECOND GRADE
}

\author{
$\mathrm{BY}$ \\ Š. MATUŠŮ
}

Mathematical Institute of the Academy of Sciences of the Czech Republic, Žitná 25, Prague 1, Czech Republic

\begin{abstract}
The existence of the classical solutions has been proved for the equations of motion for compressible fluids of second grade in a bounded domain.
\end{abstract}

1. Introduction. Geological materials and polymeric fluids are among the many substances for which the Navier-Stokes equations seem to be an inappropriate model. Many idealized material models have been suggested. The motivation for this study is to derive from the equations a fluid behaviour that is in agreement with the experimental evidence.

Nečas and his collaborators consider a stress tensor where the coefficients of viscosity are not constants but functions of the invariants of velocity fields (see [10]-[11]). Another type of model would be fluids of differential type of complexity $n$, commonly also referred to as Rivlin-Ericksen fluids. Fluids of grade $n$ form an important subclass of the fluids of complexity $n$. In such materials, only a very short part of the history of the deformation gradient has an influence on the stress. A critical review and thermodynamical analysis of the fluids of differential type can be found in the work of Dunn and Rajagopal (see $[17])$.

We investigate the steady motion of compressible fluids of second grade. Splitting our problem into three parts (Stokes problem, linearized Euler equations, and transport equation) and applying the well-known estimates, we solve three problems separately. Finally, by the Schauder theorem, we get the existence of the classical solutions assuming a sufficiently small force.

The Cauchy stress tensor $T$ is related to the motion of the constitutive equation

$$
T=-p I+\mu A_{1}+(\mu+\lambda) \operatorname{tr} A_{1} I+\alpha_{1} A_{2}+\alpha_{2} A_{1},
$$

where $p$ is the pressure, $\mu, \lambda$ are coefficients of viscosity, $\alpha_{1}, \alpha_{2}$ are material coefficients called normal stress moduli, and $A_{1}, A_{2}$ are Rivlin-Ericksen kinematical tensors which

Received June 4, 1998.

2000 Mathematics Subject Classification. Primary 76A05, 76N10, 35Q35. 
have the following form:

$$
\begin{aligned}
\left(A_{1}\right)_{i j} & =v_{i, j}+v_{j . i} \\
\left(A_{2}\right)_{i j} & =\frac{d}{d t}\left(A_{1}\right)_{i j}+L_{m i}\left(A_{1}\right)_{m j}+L_{m j}\left(A_{1}\right)_{i m} ; \\
L_{m i} & =v_{m . i} .
\end{aligned}
$$

We assume $\mu, \lambda \geq 0, \lambda \geq-\frac{2}{3} \mu, \alpha_{1}>0$ and express the divergence of the stress $T$ :

$$
\begin{aligned}
\nabla \cdot T=\mu & \Delta v+(\lambda+\mu) \nabla \nabla \cdot v \\
& +\alpha_{1}(v \cdot \nabla \Delta v+v \cdot \nabla \nabla \nabla \cdot v) \\
& +\alpha_{1}\left(\left((\nabla v)^{\mathrm{T}}: \nabla A_{1}\right)+\nabla \cdot\left(A_{1} W-W A_{1}\right)\right) \\
& +\left(\alpha_{1}+\alpha_{2}\right) \nabla \cdot A_{1}^{2}
\end{aligned}
$$

where

$$
W_{i j}=\frac{1}{2}\left(v_{i, j}-v_{j, i}\right) .
$$

The general steady motion of compressible fluids of second grade is described as follows:

$$
\left.\begin{array}{c}
\rho v \cdot \nabla v-\alpha_{1} v \cdot \nabla \Delta v-\alpha_{1} v \cdot \nabla \nabla \nabla \cdot v \\
-\mu \Delta v-(\lambda+\mu) \nabla \nabla \cdot v+\nabla p=N(v)+f \\
\nabla \cdot(\rho v)=0
\end{array}\right\} \quad \text { in } \Omega
$$

where

$$
N(v)=\alpha_{1}\left((\nabla v)^{\mathrm{T}}: \nabla A_{1}\right)+\nabla \cdot\left(A_{1} W-W A_{1}\right)+\left(\alpha_{1}+\alpha_{2}\right) \nabla \cdot A_{1}^{2},
$$

with the boundary conditions

$$
v=0 \quad \text { on } \partial \Omega
$$

while the total mass is given by

$$
\int_{\Omega} \rho=M
$$

where $\Omega \subset \mathbf{R}^{N}(N=3)$ is a bounded domain with a smooth boundary $\partial \Omega$. We are interested in the case of isothermal motion, which means that

$$
\rho=K \rho,
$$

where $K$ is a positive constant.For the sake of simplicity, we omit the third term of $(5)_{1}$.

We assume that

$$
\inf _{x \in \Omega} \rho(x)>0
$$

and we decompose the density as follows:

$$
\rho=1+\sigma .
$$

We ask whether

$$
\sup _{x \in \Omega}|\sigma(x)|<1
$$


when $\sigma$ is a small perturbation to a homogeneous state. Then problem $\left(\mathrm{P}_{1}\right)$ follows from (5) $-(7)$ :

$$
\begin{gathered}
\left.v \cdot \nabla\left(v-\alpha_{1} \Delta v\right)-\mu \Delta v=\begin{array}{r}
H-\nabla P \\
\nabla \cdot v=g
\end{array}\right\} \quad \text { in } \Omega . \\
v=0 \text { on } \partial \Omega,
\end{gathered}
$$

where

$$
\begin{gathered}
g=-\operatorname{div}(\sigma v), \quad \int_{\Omega} g=0 \\
\int_{\Omega} \sigma=0 \\
P=K \sigma+(\lambda+\mu) \operatorname{div}(\sigma v) \\
H=f+N(v)-\sigma v \cdot \nabla v
\end{gathered}
$$

Applying the Helmholtz decomposition we get

$$
\begin{gathered}
\left.\begin{array}{c}
\Delta v=w+\nabla \pi \\
\nabla \cdot v=g
\end{array}\right\} \quad \text { in } \Omega \\
v=0 \text { on } \partial \Omega
\end{gathered}
$$

with $g=-\operatorname{div}(\sigma v), \int_{\Omega} g=\int_{\Omega} \sigma=0$, and

$$
\begin{aligned}
& \left.\begin{array}{c}
\alpha_{1} v \cdot \nabla w+\mu w \nabla P=-\widetilde{H}-\alpha_{1} v \cdot \nabla \nabla \pi-\mu \nabla \pi \\
\nabla \cdot w=0
\end{array}\right\} \quad \text { in } \Omega \\
& w \cdot n=0 \quad \text { on } \partial \Omega,
\end{aligned}
$$

with

$$
\begin{aligned}
\widetilde{H} & =N(v)+\rho f-\rho v \cdot \nabla v, \\
P & =K \sigma+(\lambda+\mu) \operatorname{div}(\sigma v) .
\end{aligned}
$$

We replace $w$ in $(15)_{1}$ by $\varphi$ to have

$$
\begin{gathered}
\left.\begin{array}{c}
\Delta v=\varphi+\nabla \pi \\
\nabla \cdot v=g
\end{array}\right\} \quad \text { in } \Omega, \\
v=0 \text { on } \partial \Omega
\end{gathered}
$$

with the given data $g$ and $\varphi$. We denote the problem (16)-(19) by $\left(\mathrm{P}_{1}^{\prime}\right)$. 
How to read this problem? Problem (19) is the Stokes problem with the given data $g$ and $\varphi$. Equation (18) is a transport equation with the corresponding data $P$ and $v$, where $\operatorname{div}(\sigma v)$ is associated with $g$. The solution of (19) and the solution $\sigma$ of (18) are substituted into (16) and then the solution $w$ is associated with $\varphi$. Then we can define the mapping $F:(\varphi, g) \rightarrow(w, \operatorname{div}(\sigma v))$ for $\varphi, g$ in a suitable class. If the functions in $\varphi, g$ are smooth, then $v$ and $\pi$ are likewise smooth. Moreover. the smoothness of $P$ and $v$ (the smoothness of $v$ we know from the previous step) give us that $\sigma$ and $\operatorname{div}(\sigma v)$ are likewise smooth. As a consequence, the corresponding solution $(w, \operatorname{div}(\sigma v))=F(\varphi, g)$ to (16), (18) is also regular. We shall prove the existence of a classical solution to problem $\left(\mathrm{P}_{1}^{\prime}\right)$ and hence to problem $\left(\mathrm{P}_{1}\right)$ using the fact that the map $F$ has a fixed point in $S$.

This paper is motivated by my short visit to the University of Ferrara in July 1996 where Prof. Padula explained to me the new idea of decomposition for steady compressible fluids. Also, I would like to thank Prof. Rajagopal who actually suggested that I study this kind of problem.

2. Notation and preliminary results. Let $\Omega \subset \mathbf{R}^{3}, \partial \Omega$ of class $C^{m+2}, m \geq 0$. Let us denote by $H^{m}(\Omega)$ the Hilbert space of vector functions whose derivatives up to order $m$ belong to $L^{2}(\Omega)$ with the scalar product:

$$
(f, g)_{m}:=\sum_{|\gamma|=0}^{m}\left(D^{\gamma} f, D^{\gamma} g\right)_{0},
$$

where $(\cdot, \cdot)_{0}$ is the usual scalar product in $L^{2}(\Omega)$, and let $\|\cdot\|_{m}$ be the corresponding norm. Observe that if $m>3 / 2, H^{m}(\Omega)$ is an algebra for the pointwise multiplication of functions. Now, let us consider the closed subset of $H^{m}(\Omega)$ :

$$
X_{m}(\Omega):=\left\{\bar{g} \in H^{m}(\Omega): \nabla \cdot \bar{g}=0, \bar{g} \cdot n \mid \partial \Omega=0\right\},
$$

$n$ being the outward unit normal at $\partial \Omega$. It is not difficult to verify, by solving an elliptic Neumann problem with data in $H^{m}(\Omega)$, that the classical Helmholtz decomposition in $L^{2}(\Omega)$ carries over to $H^{m}(\Omega)$, that is, for any $\bar{g} \in H^{m}(\Omega)$ there exist two $L^{2}$-orthogonal functions $w \in X_{m}(\Omega)$ and $\pi \in H^{m+1}$ such that

$$
\bar{g}=w+\nabla \pi .
$$

The trace spaces (see [9]), associated with $H^{m}(\Omega)$, are denoted by $H^{\alpha}(\partial \Omega)$ and the norm is indicated by \|\|$_{a . \partial s 2}$.

Theorem 1 (Stokes problem). For any $\varphi \in X_{m}, g \in H^{m+1}$, Problem (19) has a unique solution $(v, \pi)$ in $H^{m+2} \times H^{m+1}$ and there exists a constant $C=C(\Omega, m, n)$ such that

$$
\|v\|_{m+2}+\|\nabla \pi\|_{m} \leq C\left(\|\varphi\|_{m}+\|g\|_{m+1}\right) .
$$

Proof. See Galdi [8].

TheOREm 2 (Transport equation). Let $m \geq-1, \partial \Omega \in C^{m+2}, \Omega$ be a bounded domain in $\mathbf{R}^{3}$ and let $v \in H^{m+2}, v \cdot n=0, P \in H^{m+1}$. Then there exists a constant $\lambda_{m}$ dependent only on $m, \partial \Omega$ such that if

$$
\lambda_{m}=\lambda_{m}\left(\|v\|_{m+2}\right)<1,
$$


then there exists just one solution of (18) with $\sigma \in H^{m+1}, \operatorname{div}(\sigma v) \in H^{m+1}$ such that

$$
\begin{gathered}
\left(1-\lambda_{m}\right)\|\sigma\|_{m+1} \leq C\|P\|_{m+1}, \\
\|\sigma\|_{m+1}+\|\operatorname{div}(\sigma v)\|_{m+1} \leq C\|P\|_{m+1} .
\end{gathered}
$$

Moreover, $\Delta \sigma \in H^{m-1}$ and the estimate

$$
\|\Delta \sigma\|_{m-1} \leq C_{1}\left(\|\Delta P\|_{m-1}+\|v\|_{m+2}\|\sigma\|_{m+1}\right)
$$

holds.

Proof. See Beraio da Veiga [3] and Novotný [12].

Theorem 3 (Euler linearized problem). Let $m \geq 3$,

$$
\begin{gathered}
\|f\|_{m} \leq \beta D, \\
\|\sigma\|_{m+1}+\|\operatorname{div}(\sigma v)\|_{m+1} \leq C_{3} \widetilde{K},
\end{gathered}
$$

where $\widetilde{K} \leq D$, and let

$$
\|v\|_{m+2}+\|\nabla \pi\|_{m} \leq C_{1} D
$$

hold for some positive $\widetilde{K}, D, C_{1}(m, \Omega), C_{3}(m, \Omega), 0<\beta<1$. Thus, if $D, \widetilde{K}$ satisfy

$$
\begin{gathered}
D \leq \min \left\{\frac{\mu}{\left|\alpha_{1}\right| C_{1} C_{2}}, \frac{\mu-\left(\mu C_{1} C_{2}-C_{3} C_{2} \beta \widetilde{K}\right)}{C_{1} C_{2}\left[C_{1} a+C_{3} C_{1} \widetilde{K}+\left|\alpha_{1}\right| C_{1}+\left|\alpha_{1}\right| C_{2}\right]}\right\}, \\
\widetilde{K} \geq \gamma,
\end{gathered}
$$

which will be defined in the proof and $a=\max \left\{\left|\alpha_{1}\right|,\left|\alpha_{1}+\alpha_{2}\right|\right\},\left\{\left|\alpha_{1}\right|,\left|\alpha_{1}+\alpha_{2}\right|\right\} \geq$ $\frac{\sqrt{\mu 4 C_{1} C_{3} C_{2}\left(C_{1} C_{2}-1\right)}+C_{3} C_{2} \beta}{2 C_{1}^{2} C_{2}+C_{2}^{2} C_{1}}$, then Problem (16) has a unique solution $w \in X_{m}(\Omega)$ satisfying

$$
\|w\|_{m} \leq D
$$

Proof. Applying the operator $D^{\alpha}$ to $(16)_{1}, 0 \leq \alpha \leq m$, and taking the $L^{2}$ scalar product with $D^{\alpha} w$ and adding the resulting identities we get

$$
\begin{aligned}
\mu\|w\|_{m}^{2}+\alpha_{1}(v \cdot \nabla w, w)_{m}= & -(\widetilde{H}, w)_{m}+(\nabla P, w)_{m} \\
& -\alpha_{1}(v \cdot \nabla \pi, w)_{m}-\mu(\nabla \pi, w)_{m} .
\end{aligned}
$$

Taking into account that $N(v)$ is a sum of products of first and second derivatives of $v$, the Schwarz inequality and the algebra property of $H^{m}(\Omega)$ yield

$$
\|N(v)\|_{m} \leq C a\|v\|_{m+2}^{2} \text {. }
$$

We apply the divergence operator on $(16)_{1}$. In this way, we come to consider the Neumann problem:

$$
\begin{aligned}
& \Delta P=\operatorname{div}\left(\widetilde{H}+\alpha_{1} v \cdot \nabla \nabla \pi+\mu \nabla \pi+\alpha_{1} v \cdot \nabla w\right)=\widetilde{F} \\
& \frac{\partial P}{\partial n}=(\widetilde{H}+\nabla \pi) \cdot n=\widetilde{G} .
\end{aligned}
$$


From well-known results on the regularity of (34) we have the following estimate:

$$
\|\nabla P\|_{m} \leq C\left(\|\widetilde{F}\|_{m-1}+\|\widetilde{G}\|_{m-1 . \partial \Omega}\right)
$$

Then

$$
\begin{aligned}
(\nabla P, w)_{m} \leq C & \left(\|\tilde{H}\|_{m}+\left|\alpha_{1}\right|\|v\|_{m+2}\|\nabla \pi\|_{m}\right. \\
& \left.+\mu\|\nabla \pi\|_{m}+\left|\alpha_{1}\right|\|v\|_{m+2}\|w\|_{m}\right)\|w\|_{m} \\
\leq C & \left(a\|v\|_{m+2}^{2}+\|\sigma\|_{m+1}\|f\|_{m}+\|\sigma\|_{m+1}\|v\|_{m+2}^{2}\right. \\
& \left.\quad+\left|\alpha_{1}\right|\|v\|_{m+2}\|\nabla \pi\|_{m}+\mu\|\nabla \pi\|_{m}+\left|\alpha_{1}\right|\|v\|_{m+2}\|w\|_{m}\right)\|w\|_{m}
\end{aligned}
$$

Under assumptions (27)-(29), this implies that

$$
\begin{aligned}
\|\nabla P\|_{m} \leq C\left(a C_{1}^{2} D^{2}+C_{3} \tilde{K} \beta D+\right. & C_{3} \widetilde{K} C_{1}^{2} D^{2}+\left|\alpha_{1}\right| C_{1}^{2} D^{2} \\
& \left.+\mu C_{1} D+\left|\alpha_{1}\right| C_{1} D\|w\|_{m}\right) .
\end{aligned}
$$

Similarly as in [7] we estimate the following terms:

$$
\left|(v \cdot \nabla \nabla \pi, w)_{m}\right| \leq C\|v\|_{m+2}\|\nabla \pi\|_{m}\|w\|_{m}
$$

and

$$
\left|(v \cdot \nabla w, w)_{m}\right| \leq C\|v\|_{m+2}\|w\|_{m}^{2} .
$$

Collecting (33), (36), (35)', (35)" and (27)-(29) we obtain that

$$
\begin{aligned}
\left(\mu-C_{2} C_{1}\left|\alpha_{1}\right| D\right)\|w\|_{m} \leq C_{2} & \left(a C_{1}^{2} D^{2}+C_{3} \tilde{K} \beta D\right. \\
& \left.+C_{3} \widetilde{K} C_{1}^{2} D^{2}+\left|\alpha_{1}\right| C_{1}^{2} D^{2}+\mu C_{1} D\right) .
\end{aligned}
$$

It follows that

$$
\begin{gathered}
\|w\|_{m} \leq C_{2} D\left[a C_{1}^{2} D+C_{3} \beta \widetilde{K}+C_{3} C_{1}^{2} \widetilde{K} D+\left|\alpha_{1}\right| C_{1}^{2} D\right. \\
\left.+\mu C_{1}\right]\left(\mu-\left|\alpha_{1}\right| C_{1} C_{2} D\right)^{-1} .
\end{gathered}
$$

If $D$ satisfies condition (30), then

$$
\|w\|_{m} \leq D
$$

We need that $\tilde{K} \leq D$. This implies firstly that

$$
\widetilde{K} \leq \frac{\mu}{\left|\alpha_{1}\right| C_{1} C_{2}}
$$

From the second assumption, which we have on $D$, it follows that

$$
\widetilde{K} \leq \frac{\mu-\left(\mu C_{1} C_{2}-C_{2} C_{3} \beta \widetilde{K}\right)}{C_{1} C_{2}\left[C_{1} a+C_{3} C_{1} \widetilde{K}+\left|\alpha_{1}\right| C_{1}+\left|\alpha_{1}\right| C_{2}\right]} .
$$

This implies, firstly, the condition on $\left|\alpha_{1}\right|,\left|\alpha_{1}+\alpha_{2}\right|$ :

$$
\left\{\left|\alpha_{1}\right|,\left|\alpha_{1}+\alpha_{2}\right|\right\} \geq \frac{\sqrt{4 \mu C_{1} C_{2} C_{3}\left(C_{1} C_{2}-1\right)}+C_{3} C_{2} \beta}{2 C_{1}^{2} C_{2}+C_{2}^{2} C_{1}},
$$

secondly, the condition on $C_{1}$ :

$$
C_{1} C_{2} \geq 1
$$


and thirdly that

$$
\gamma=\frac{-\left[C_{2} C_{1}^{2} a+\left|\alpha_{1}\right| C_{1}^{2} C_{2}+\left|\alpha_{1}\right| C_{2}^{2} C_{1}-C_{3} C_{2} \beta\right]+\sqrt{D_{1}}}{2 C_{1}^{2} C_{3} C_{2}},
$$

where

$$
\begin{aligned}
D_{1}=[ & \left.C_{2} C_{1}^{2} a+\left|\alpha_{1}\right| C_{1}^{2} C_{2}+\left|\alpha_{1}\right| C_{2}^{2} C_{1}-C_{3} C_{2} \beta\right]^{2} \\
& -4 C_{1}^{2} C_{3} C_{2}\left(\mu C_{1} C_{2}-\mu\right) .
\end{aligned}
$$

Thus we have an a priori estimate. Now, we apply the Galerkin method. We define the orthonormal set of functions in $X_{m}$ by

$$
\psi_{m} \in X_{2 m}(\Omega)
$$

and solve the eigenvalue problem

$$
\left(\psi_{k}, w\right)_{m}=\lambda_{k}\left(\psi_{k}, w\right)_{0} \quad \forall w \in X_{m}(\Omega) .
$$

We define $X$ as the closure of the functions $\left(\psi_{1}, \ldots, \psi_{\nu}\right)$ in $X_{m}$ and $\widetilde{P}$ as the projection from $X_{m}$ onto $X$. For any $w \in X$ we can write

$$
w^{(\nu)}=\sum_{j=1}^{\nu} g_{j} v \psi_{j}, \quad g_{i \nu} \in \mathbf{R} .
$$

Thus, we have to solve the nonlinear algebraic system

$$
\begin{aligned}
& \mu\left(w^{(\nu)}, \psi_{k}\right)+\left(\widetilde{P}\left(v \cdot \nabla w^{(\nu)}\right), \psi_{k}\right)_{0} \\
& \quad=\left(\widetilde{P}(v \cdot \nabla \nabla \pi), \psi_{k}\right)_{0}+\left(\widetilde{P} \widetilde{H}, \psi_{k}\right)_{0}-\left(\widetilde{P} \nabla P, \psi_{k}\right)_{0} .
\end{aligned}
$$

Now, using the standard method, we obtain the existence of a solution to the algebraic system (48) for any $\nu>0$ under assumptions (30). Since (39) is a uniform estimate it follows that $\left\{w^{(\nu)}\right\}_{\nu \in N}$ is uniformly bounded in $X_{m}(\Omega)$ and this is sufficient for the existence of a unique solution $w \in X_{m}(\Omega)$ to Problem (46).

Now, we want to prove that $\varphi=w$ and $g=\operatorname{div}(\sigma v)$. We apply the Schauder fixed point theorem in the following form.

LEMma. Let $S$ be a closed convex set in a Banach space $B$ and let $F$ be an absolutely continuous operator from $S$ into $B$ such that $F(S) \subset S$. Then $F$ has a fixed point in $S$.

Proof. See Friedman [6].

We put

$$
B=X_{m-1} \times H^{m}(\Omega), \quad m \geq 1
$$

and for $D>0$

$$
S=S_{D}=\left\{(\varphi, g) \in X_{m-1}(\Omega) \times H^{m},\|\varphi\|_{m} \leq D,\|g\|_{m+1} \leq D\right\} .
$$

Theorem 4. Let $m \geq 3, D, \widetilde{K}$ satisfy (30), (31) and (*) which we will define later. Then the boundary value problem has a unique solution $(v, \rho)$ such that $\rho=1+\sigma, v \in H^{m+2}$, $\sigma \in H^{m+1}$, and verifying the estimate

$$
\|v\|_{m+2}+\|\sigma\|_{m+1} \leq C_{1} D
$$


Proof. First of all we prove that the map $F$ assigns to every $(\varphi, g) \in S_{D}$ the unique function $(w, \operatorname{div}(\sigma v))=F((\varphi, g))$ with $w$ being the solution of Problem (16) and $\operatorname{div}(\sigma v)$ the solution of Problem (18). The map $F$ is well defined by Theorems $1-3$. Moreover, if $D, \widetilde{K}$ satisfy (30), (31), (*) with $(40)-(43)^{\prime},(44)$, then this means that $F\left(S_{D}\right) \subset S_{D}$. Furthermore, the compact embedding of $X_{m}(\Omega)$ in $X_{m-1}$ implies that every sequence of functions uniformly bounded in $X_{m}(\Omega)$ is strongly convergent in $X_{m-1}(\Omega)$ and the same follows from the compact embedding of $H^{m+1}(\Omega)$ in $H^{m}(\Omega)$. Then we only have to check the continuity of $F$. It is not difficult to prove that

$$
\left\|N\left(v_{1}\right)-N\left(v_{2}\right)\right\|_{m} \leq C a\left(\left\|v_{1}\right\|_{m+2}+\left\|v_{2}\right\|_{m+2}\right) \times\left(\left\|v_{1}\right\|_{m+2}-\left\|v_{2}\right\|_{m+2}\right) .
$$

Let now $\left\{\varphi_{n}, g_{n}\right\}_{n \in N}$ be a sequence in $S_{D}$ such that $\left(\varphi_{n}, g_{n}\right) \rightarrow(\varphi, g) \in S_{D}$ as $n \rightarrow+\infty$, and let $\left(v_{n}, \pi_{n}\right)$ be the solution to Problem (19) with the corresponding data $\left(\varphi_{n}, g_{n}\right)$. As a result of Theorem $1,\left(v_{n}, \nabla \pi_{n}\right)$ converges in $H^{m+1}(\Omega) \times H^{m-1}(\Omega)$ to $(v, \nabla \pi)$, where $(v, \nabla \pi)$ is the solution of (19) with the corresponding data $(\varphi, g)$. As a result of Theorem $2,\left(\sigma_{n}, \operatorname{div}\left(\sigma_{n} v_{n}\right)\right)$ converges in $H^{m}(\Omega) \times H^{m}(\Omega)$ to $(\sigma, \operatorname{div}(\sigma v))$, where $(\sigma, \operatorname{div}(\sigma v))$ is the solution of (18) with the corresponding data $P, v$, where $P$ depends on $v, \pi, w$. Let $\left\{w_{n}\right\}$ be the solution of Problem (16) and $\{\sigma, \operatorname{div}((\sigma v))\}$ be the solution of (18). We take the difference of the two solutions $w-w_{n}$ and apply $D^{\alpha-1}, 0 \leq \alpha \leq m$. Using $\nabla \cdot w=0$, $w \cdot n=0$, multiplying by $D^{\alpha-1}\left(w-w_{n}\right)$ and adding the resulting identities we get, after integration by parts,

$$
\begin{aligned}
\mu\left\|w-w_{n}\right\|_{m-1}^{2}= & -\alpha_{1} \int_{\Omega} D^{\alpha-1}\left(\left(v-v_{n}\right) \cdot \nabla w\right) D^{\alpha-1}\left(w-w_{n}\right) \\
& -\alpha_{1} \int_{\Omega}\left(D^{\alpha-1}\left(v-v_{n}\right) \cdot \nabla \nabla \pi\right) D^{\alpha-1}\left(w-w_{n}\right) \\
& -\alpha_{1} \int_{\Omega} D^{\alpha-1}\left(v_{n} \cdot \nabla \nabla\left(\pi_{n}-\pi\right)\right) D^{\alpha-1}\left(w-w_{n}\right) \\
& -\alpha_{1} \int_{\Omega} D^{\alpha-1}\left(v_{n} \cdot \nabla\left(w_{n}-w\right)\right) D^{\alpha-1}\left(w-w_{n}\right) \\
& -\int_{\Omega} D^{\alpha-1}\left(N(v)-N\left(v_{n}\right)\right) D^{\alpha-1}\left(w-w_{n}\right) \\
& +\int_{\Omega} D^{\alpha-1}\left(\nabla\left(P-P_{n}\right)\right) D^{\alpha-1}\left(w-w_{n}\right) \\
& +\int_{\Omega} D^{\alpha-1}\left(\left(\sigma-\sigma_{n}\right) v \cdot \nabla v\right) D^{\alpha-1}\left(w-w_{n}\right) \\
& +\int_{\Omega} D^{\alpha-1}\left(\sigma_{n}\left(v-v_{n}\right) \cdot \nabla v\right) D^{\alpha-1}\left(w-w_{n}\right) \\
& +\int_{\Omega} D^{\alpha-1}\left(\sigma_{n} v_{n} \nabla\left(v-v_{n}\right)\right) D^{\alpha-1}\left(w-w_{n}\right) \\
& -\int_{\Omega} D^{\alpha-1}\left(\left(\sigma-\sigma_{n}\right) f\right) D^{\alpha-1}\left(w-w_{n}\right) . \\
&
\end{aligned}
$$

In Problem (18) we have

$$
\left\|\sigma-\sigma_{n}\right\|_{m}+\left\|\operatorname{div}(\sigma v)-\operatorname{div}\left(\sigma_{n} v_{n}\right)\right\|_{m} \leq\left\|\left(P-P_{n}\right)\right\|_{m} .
$$


Applying the well-known regularity results on the Neumann problem $\Delta\left(P_{n}-P\right)=$ $\widetilde{F}_{1}, \frac{\partial}{\partial n}\left(P_{n}-P\right)=\widetilde{G}_{1}$ as in Theorem 3 on Problem $\Delta P=\widetilde{F}, \frac{\partial P}{\partial n}=\widetilde{G}$ and applying the Sobolev embedding we get

$$
\begin{aligned}
\| \sigma-\sigma_{n} & \left\|_{m}+\right\| \operatorname{div}(\sigma v)-\operatorname{div}\left(\sigma_{n} v_{n}\right) \|_{m} \\
\leq & C\left\|\nabla\left(P-P_{n}\right)\right\|_{m-1} \leq C\left(\|\widetilde{F}\|_{m-2}+\|\widetilde{G}\|_{m-2, \partial \Omega}\right) \\
\leq & C\left(\left\|v-v_{n}\right\|_{m+2}\|w\|_{m-1}+\left\|v-v_{n}\right\|_{m+2}\|\nabla \pi\|_{m}\right. \\
& +\left\|v_{n}\right\|_{m+2}\left\|\nabla\left(\pi-\pi_{n}\right)\right\|_{m}+\left|\alpha_{1}\right|\left\|v_{n}\right\|_{m+2}\left\|w-w_{n}\right\|_{m} \\
& +a\left(\|v\|_{m+2}+\left\|v_{n}\right\|_{m+2}\right)\left\|v-v_{n}\right\|_{m+2}+\left\|\sigma-\sigma_{n}\right\|_{m}\|v\|_{m+2}^{2} \\
& +\left\|\sigma_{n}\right\|_{m}\left\|v-v_{n}\right\|_{m+2}\|v\|_{m+2}+\left\|\sigma_{n}\right\|_{m}\left\|v_{n}\right\|_{m+2}\left\|v-v_{n}\right\|_{m+2} \\
& \left.\quad+\left\|\sigma-\sigma_{n}\right\|_{m}\|f\|_{m-1}\right) \\
\leq & \widetilde{C}\left(\left\|\varphi-\varphi_{n}\right\|_{m}+\left\|g-g_{n}\right\|_{m+1}+\left|\alpha_{1}\right|\left\|v_{n}\right\|_{m+2}\left\|w-w_{n}\right\|_{m-1}\right) .
\end{aligned}
$$

From (53) it follows that

$$
\begin{gathered}
\left(\left(v-v_{n}\right) \cdot \nabla w, w-w_{n}\right)_{m-1} \leq C\left(\left\|v-v_{n}\right\|_{m+2}\|w\|_{m-1}\left\|w-w_{n}\right\|_{m-1}\right) \\
\left(\left(v-v_{n}\right) \nabla \nabla \pi, w-w_{n}\right)_{m-1} \leq C\left(\left\|v-v_{n}\right\|_{m+2}\|\pi\|_{m+1}\left\|w-w_{n}\right\|_{m-1}\right), \\
\left(v_{n} \cdot \nabla \nabla\left(\pi_{n}-\pi\right), w-w_{n}\right)_{m-1} \leq C\left\|v_{n}\right\|_{m+2}\left\|\pi_{n}-\pi\right\|_{m+1}\left\|w-w_{n}\right\|_{m-1}, \\
\left(v_{n} \cdot \nabla\left(w_{n}-w\right), w-w_{n}\right)_{m-1} \leq C\|v\|_{m+2}\left\|w_{n}-w\right\|_{m-1}^{2} \\
\left(N(v)-N\left(v_{n}\right), w-w_{n}\right)_{m-1} \leq C\left(\|v\|_{m+2}+\left\|v_{n}\right\|_{m+2}\right)\left\|v-v_{n}\right\|_{m+2}\left\|w-w_{n}\right\|_{m-1}, \\
\left(\left(\sigma-\sigma_{n}\right) f, w-w_{n}\right)_{m-1} \leq C\left\|\sigma-\sigma_{n}\right\|_{m}\|f\|_{m-1}\left\|w-w_{n}\right\|_{m-1} \\
\left(\left(\sigma-\sigma_{n}\right) v \cdot \nabla v, w-w_{n}\right) \leq C\|v\|_{m+2}^{2}\left\|w-w_{n}\right\|_{m-1}\left\|\sigma-\sigma_{n}\right\|_{m} \\
\left(\sigma_{n}\left(v-v_{n}\right) \nabla v, w-w_{n}\right) \leq C\left\|\sigma_{n}\right\|_{m-1}\left\|v-v_{n}\right\|_{m+2}\|v\|_{m+2}\left\|w-w_{n}\right\|_{m-1} \\
\left(\sigma_{n} v_{n} \nabla\left(v-v_{n}\right), w-w_{n}\right) \leq C\left\|v_{n}\right\|_{m+2}\left\|\sigma_{n}\right\|_{m}\left\|v-v_{n}\right\|_{m+2}\left\|w-w_{n}\right\|_{m-1}
\end{gathered}
$$

Applying Theorems 1 and 3 we then get

$$
\begin{array}{r}
\left\|w-w_{n}\right\|_{m-1}\left(\mu-\left|\alpha_{1}\right| C\|v\|_{m+2}\right)+\left\|\operatorname{div}\left(\sigma^{n} v^{n}\right)-\operatorname{div}(\sigma v)\right\|_{m} \\
+\left\|\sigma-\sigma_{n}\right\|_{m}\left(1-C_{2} \beta D-C_{2} C_{1}^{2} D^{2}\right) \\
\leq C\left(\left\|\varphi-\varphi_{n}\right\|_{m}+\left\|g-g_{n}\right\|_{m+1}\right) .
\end{array}
$$

We assume that

$$
D<\frac{\mu}{\left|\alpha_{1}\right| C C_{1}}, \quad D<p
$$

where

$$
p=\frac{-C_{2} \beta+\sqrt{C_{2}^{2} \beta^{2}+4 C_{2} C_{1}^{2}}}{2 C_{2} C_{1}^{2}}
$$


and set $h=\mu-C C_{1} D$. Then

$$
\begin{gathered}
\left\|w-w_{n}\right\|_{m-1}+\left\|\operatorname{div}\left(\sigma^{n} v^{n}\right)-\operatorname{div}(\sigma v)\right\|_{m} \\
\leq \frac{c}{h}\left(\left\|\varphi-\varphi_{n}\right\|_{m}+\left\|g-g_{n}\right\|_{m+1}\right) .
\end{gathered}
$$

Then $w-w_{n} \in X_{m-1}, \operatorname{div}\left(\sigma^{n} v^{n}\right)-\operatorname{div}(\sigma v) \in H^{m}$, the compactness of $F\left(S_{D}\right)$ in $X_{m-1} \times$ $H^{m}$, a "subsequence argument", and the uniqueness of the limit therefore imply that $F$ is continuous in the topology of $X_{m-1}(\Omega) \times H^{m}$ for $m \geq 3$.

Acknowledgment. The present research has been supported under Grant No. 201/96/0313 of the Grant Agency of the Czech Republic.

\section{REFERENCES}

[1] R. A. Adams, Sobolev Spaces, Academic Press, New York, 1975

[2] S. Agmon, A. Douglis, and L. Nirenberg, Estimates near the boundary for solutions of elliptic partial differential equations satisfying general boundary conditions, Comm. Pure Appl. Math. 17, 35-92 (1964)

[3] H. Beraio da Veiga, Boundary value problems for a class of first order partial differential equations in Sobolev spaces and applications to the Euler flow, Rend. Sem. Mat. Univ. Padova 79, 247-273 (1988)

[4] L. Cattabriga, Su un problema el contorno relativo al sistema di equanioni di Stokes, Rend. Math. Sem. Univ. Padova 31, 308-340 (1961)

[5] J. E. Dunn and R. L. Fosdick, Thermodynamics, stability, and boundedness of fluids of complexity 2 and fluids of second grade, Arch. Rational Mech. Anal. 56, 191-252 (1974)

[6] A. Friedman, Partial Differential Equations, Holt, Rinehard, and Winston, Inc., New York, 1969

[7] G. P. Galdi and V. Coscia, Existence, uniqueness and stability of regular steady motions of a second grade fluid, Internat J. Nonlinear Mech. 29, 493-506 (1994)

[8] G. P. Galdi, An Introduction to the Mathematical Theory of the Navier-Stokes Equations I, II, Springer-Verlag, New York, 1994

[9] J.-L. Lions and E. Magenes, Nonhomogeneous boundary value problems, Vol. I, Springer-Verlag, Berlin, Heidelberg, New York, 1972

[10] J. Málek, J. Nečas, and M. Rǔžčka, On the non-Newtonian incompressible fluids, Mathematical Methods and Models in Applied Sciences, Vol. 3, 35-63 (1993)

[11] J. Nečas, Theory of multipolar viscous fluids, The Mathematics of Finite Elements and Applications, (Ed. J. R. Whiteman), Academic Press, London, 1991, pp. 233-244

[12] A. Novotný, About steady transport equations $I-L^{p}$-approach in domains with smooth boundaries, Comment. Math. Univ. Carolina 37, 43-89 (1996)

[13] M. Padula, Recent contributions and open questions in the mathematical theory of steady motions of viscous compressible fluids, Preprint, 1996

[14] K. R. Rajagopal, personal communications

[15] R. S. Rivlin and J. L. Ericksen, Stress-deformation relations for isotropic materials, J. Rational Mech. Anal. 4, 323-425 (1955)

[16] K. Temam, On the Euler equations of incompressible perfect fluids, J. Funct. Anal. 20, 32-43 (1975)

[17] J. E. Dunn and K. R. Rajagopal, Fluids of differential type: Critical review and thermodynamic analysis, Internat. J. Engrg. Sci. 33, 689-729 (1995) 